\title{
ASPECTOS SOCIODEMOGRÁFICOS E REPRODUTIVOS DO ABORTAMENTO INDUZIDO DE MULHERES INTERNADAS EM UMA
}

\author{
Socio demographic and reproductive aspects of the induced \\ miscarriage of women hospitalized in a maternity hospital of serra, \\ state of Espírito Santo
}

Aspectos socio demográficos y reproductivos del aborto inducido en mujeres internadas en una maternidad del municipio de Sierra, ES.

\author{
Priscilla Rocha Araujo Nader ${ }^{1}$ \\ Angélica Espinosa Miranda ${ }^{3}$
}

Cristina Ribeiro Macedo 2

Ethel Leonor Nóia Maciel ${ }^{4}$

\section{RESUMO}

Objetivo: Comparar aspectos sociodemográficos e reprodutivos entre mulheres que induziram a interrupção da gestação e mulheres que levaram a gestação a termo, admitidas em uma maternidade pública. Métodos: Estudo caso-controle onde foram incluídas 21 mulheres que realizaram abortamento induzido e 83 que tiveram gestação a termo, no período de agosto de 2005 a janeiro de 2006. Resultados: Não houve diferença significativa na comparação entre as freqüências dos grupos em relação à idade, cor da pele, anos de estudo, religião, renda mensal, idade da menarca, idade da coitarca, idade da primeira gestação, número de gestações, intervalo entre a última e a penúltima gestação e o número de filhos vivos. 0 estado conjugal casada/em união estável $[\mathrm{OR}=0,241$ (IC95\% 0,061-0,951)] e desejo pela gravidez $[\mathrm{OR}=0,168$ (IC95\% 0,042 - 0,669)] mostraram-se associados à ocorrência de abortamento induzido como fatores de proteção. Conclusão: Para atenuar o problema do abortamento inseguro, deve-se investir na promoção da saúde reprodutiva incentivando o acesso à educação sexual e ao planejamento familiar.

Palavras-chave: Abortamento. Planejamento Familiar. Saúde. Saúde materno-infantil.

\begin{abstract}
Goal: Comparing socio demographic and reproductive aspects between women who had induced the interruption of pregnancy and women who had taken the pregnancy period in full hospitalized in a public maternity. Methods: Study case-control that 21 women who had an induced miscarriage and 83 who had the full pregnancy period were included in the period from August, 2005 to January, 2006. Results: There was no significant difference in the comparison between the frequency of groups related to age, skin color, study years, religion, monthly income, first-period age, first sexual-relation age, first pregnancy age, number of pregnancy, gap between last and the one before the last pregnancy and the number of live children. The marital status married/ stable union $[\mathrm{OR}=0,241(\mathrm{IC95 \%} 0,06-0,951)]$ and the pregnancy will $[0 R=0,168($ IC95\% 0, $042-0,669)]$ are associated to the occurrence of induced miscarriage as a protection factor. Conclusions: To make evident the unsafe miscarriage problem, there must be an investment in the promotion of the reproductive health motivating the access to the sexual education and the family planning.
\end{abstract}

Keywords: Miscarriage. Family planning. Health. Maternal and Child Health.

\section{Resumen}

Objetivo: Comparar aspectos socio demográficos y reproductivos entre mujeres que indujeron la interrupción del embarazo y mujeres que concluyeron el embarazo y fueron admitidas en una maternidad pública. Métodos: Estudio casocontrol donde fueron incluidas 21 mujeres que realizaron aborto inducido y 83 que tuvieron la gestión completa, en el período de agosto de 2005 a enero de 2006. Resultados: No hubo diferencia significativa en la comparación entre las frecuencias de los grupos en relación a la edad, color de piel, años de estudio, religión, renta mensual, edad de menopausia, edad de iniciación sexual, edad del primer embarazo, número de embarazos, intervalo entre el último y el penúltimo embarazo y el número de hijos vivos. El estado conyugal casado/a estable $[O R=0,241($ IC95\% 0,061-0,951)] y deseo por el embarazo [OR=0,168 (IC95\% 0,042-0,669)] se muestran asociados al hecho del aborto inducido como factores de protección. Conclusión: Para atenuar el problema del aborto inseguro, se debe invertir en la promoción de la salud reproductiva incentivando el acceso a la educación sexual y a la planificación familiar.

Palabras-claves: Aborto. Planificación Familiar. Salud. La Salud Materna e Infantil 


\section{INTRODUÇ̃̃O}

As gestações não planejadas e os abortamentos provocados são atribuídos, em grande parte, ao atraso cultural, à paternidade irresponsável, ao uso inadequado de métodos anticoncepcionais, à falta de educação sexual, ao problema da gravidez na adolescência. Esses fatores vão desde questões relacionadas com a precariedade da situação pessoal e de condições de vida diante da urbanização desenfreada até as carências de programas educativos e de planejamento reprodutivo'.

0 abortamento é considerado crime pela lei brasileira, entretanto, ele é freqüentemente praticado e dificilmente a punição judicial é aplicada. Por outro lado, os casos de abortamento permitidos pela lei são relativamente pouco praticados na condição de abortamento legal, seja por falta de acesso aos serviços de saúde, seja pelo medo de incriminação e desconhecimento dos trâmites legais para realizá-lo².

As restrições legais não são solução para o problema do abortamento no país, e a realização clandestina dessa prática está associada a grandes riscos para a saúde física e mental das mulheres, muitas vezes causando mortes maternas, que, na maioria das vezes, poderiam ser prevenidas ${ }^{3}$.

Diante da constatação de que essas mortes e danos à saúde das mulheres são evitáveis, duas importantes questões precisam ser consideradas: a descriminalização do aborto ${ }^{4} \mathrm{e}$ a criação de serviços seguros para assistência ao abortamento, uma vez que essas iniciativas podem ajudar a salvar a vida das mulheres e também diminuir os custos, para o sistema, relacionados com as complicações do abortamento inseguro ${ }^{5}$.

Dallari ${ }^{6}$ reflete que toda a sociedade e, em particular, aqueles que trabalham direta ou indiretamente com a saúde da população devem debater em busca do consenso possível sobre a prática do abortamento, a fim de evitar a arbitrariedade ética e encontrar a melhor maneira para lidar com o problema.

Durante a realização de um estudo transversal para avaliar as características de abortamentos induzidos e espontâneos atendidos em uma maternidade pública ${ }^{7}$, concluiu-se que havia diferenças significativas entre os grupos abortamento induzido e abortamento espontâneo, em relação ao planejamento da gestação, desejo pela gravidez, pensamento sobre abortar, recebimento de conselho para abortar e tratamento com antibiótico no período da internação. As diferenças encontradas permearam pontos como planejamento e desejo pela gravidez e nos motivaram a realização da atual pesquisa, pela seguinte inquietação: que fatores, entre aquelas mulheres de mesma classe social e mesma faixa etária, determinavam a escolha entre levar uma gestação a termo ou interrompê-la?

Diante do exposto, este estudo teve como objetivo comparar aspectos sociodemográficos e reprodutivos entre mulheres que induziram a interrupção da gestação e mulheres que levaram a gestação a termo admitidas em uma maternidade pública.

\section{MATERIAIS E MÉTODOS}

\section{Delineamento do estudo}

Trata-se de um estudo caso-controle entre mulheres admitidas em uma maternidade pública do Município da Serra, Espírito Santo, Brasil.

\section{Coleta de Dados}

A pesquisa foi desenvolvida na Maternidade de Carapina, instituição pública onde são realizados $48,8 \%$ dos partos do município da Serra-ES ${ }^{8}$. A maternidade presta assistência a gestantes, parturientes, puérperas e recém-nascidos de baixo risco.

\section{Definição de casos e controles}

Mulheres admitidas no serviço, no período de agosto de 2005 a janeiro de 2006, foram selecionadas para participar do estudo, sendo alocadas em dois grupos: (1) Grupo-caso: 21 mulheres admitidas na maternidade por complicações do abortamento e que declararam tê-lo induzido. De acordo com os critérios estabelecidos pela $\mathrm{OMS}^{9}$, consideraram-se abortamentos induzidos aqueles que se encaixaram na categoria "aborto seguramente provocado", identificados a partir da declaração da mulher; (2) Grupo-controle: 83 mulheres que tiveram gestação a termo, selecionadas aleatoriamente da seguinte maneira: foram as quatro primeiras internações após a escolha de uma mulher do grupo-caso.

\section{Cálculo de tamanho da amostra}

Utilizando os procedimentos de tamanho de amostra para um estudo caso-controle não pareado, precisão desejada de $80 \%$, percentual de desejo pela gravidez no grupo dos controles (gestação a termo) de $60 \%$ e no grupo dos casos (aborto induzido) de $20 \%$ para um nível de significância de $5 \%$, em uma proporção de 4:1, o tamanho da amostra para determinar diferenças entre os grupos foi de 17 casos e 68 controles.

Definimos o plano amostral elegendo os cinco dias úteis da semana para realizar as entrevistas. Como todas as mulheres que se internaram com diagnóstico de abortamento no período selecionado para a coleta de dados foram entrevistadas com o objetivo de identificar os possíveis casos, a amostra do estudo constituiu-se de 21 casos e 83 controles.

\section{Definição das variáveis}

Foi construído, para levantamento das variáveis, um formulário subdividido em três blocos - Bloco l: características sociodemográficas; Bloco ll: características reprodutivas; Bloco III: informações sobre o pai da criança e familiares - contendo perguntas abertas e fechadas. Esse instrumento baseou-se nas variáveis da pesquisa realizada por Fonseca et al..$^{10}$.Foi realizado um estudo piloto para testar a logística do instrumento no mês de julho de 2005.

\section{Análise dos dados}

Para análise dos dados foi utilizado o Social Package Statistical Science (SPSS) - versão 14.0 (2006). Diferenças 
entre proporções foram avaliadas através do teste de quiquadrado e do teste exato de Fisher para as caselas com valores $<5$, com níveis de significância de $5 \%$. Foi calculada a razão de chance (OR) com um intervalo de confiança (IC) de $95 \%$. As variáveis que apresentaram $p$-valor $<0,10$ foram incluídas nas análises multivariadas subseqüentes - regressão logística multivariada, não condicional, hierarquizada.

\section{Considerações éticas}

Atendendo a resolução 196/96 do Ministério da Saúde, este projeto foi aprovado pelo Comitê de Ética em Pesquisa da Universidade Federal do Espírito Santo sob o número 025/05 e autorizado pela direção da Maternidade de Carapina. As participantes foram incluídas no estudo após a assinatura do Termo de Consentimento Livre e Esclarecido, sendo respeitada a participação voluntária na pesquisa e garantindo às mesmas 0 anonimato de seus depoimentos.

Tomou-se o cuidado de realizar as entrevistas, nos casos que tiveram indicação de curetagem, sempre após 0 procedimento, levando-se em conta que antes da referida intervenção a mulher encontra-se, de maneira geral, sangrando, com dor e ansiosa, o que poderia acarretar desconforto por parte da entrevistada.

\section{RESULTADOS}

Durante o período estudado foram abordados 83 possíveis casos; destes, apenas 21 declararam o abortamento como sendo induzido.

Não houve diferença significativa na comparação entre as freqüências dos grupos em relação à idade, cor da pele, anos de estudo, religião e renda mensal (Tabela 1).

Entre as mulheres que admitiram ter induzido o abortamento, somente $23,8 \%$ se declararam casadas ou viviam em união estável, $23,8 \%$ disseram ter namorado, e, ao somarmos as categorias separada/divorciada e solteira, concluise que $52,4 \%$ dessas mulheres não mantinham qualquer tipo de relacionamento afetivo com o pai da criança. As diferenças entre os grupos foram estatisticamente significativas (Tabela 1). Ao se avaliar a razão de chances entre as casadas ou em união estável e as não casadas dos dois grupos, conclui-se que a indução do abortamento recebe uma proteção de $93 \%$ quando a mulher é casada ou vive em união estável. Um número maior de mulheres do grupo abortamento induzido, quando comparado com o grupo-controle, trabalha fora, totalizando $62,0 \%$ das mulheres que induziram o abortamento. A ocupação como dona de casa foi predominante no grupo-controle $(63,9 \%)$. Quando o grupo-controle foi correlacionado com o grupo abortamento induzido, os resultados demonstraram diferenças estatisticamente significantes. As mulheres que induziram o abortamento tiveram 7,5 vezes mais chances de trabalhar fora, estudar ou ter como objetivo conseguir um emprego do que aquelas que se dizem donas de casa (Tabela1).

\begin{tabular}{|c|c|c|c|c|c|c|}
\hline \multirow[b]{3}{*}{ Caracteristicas } & \multirow{2}{*}{\multicolumn{2}{|c|}{$\begin{array}{l}\text { Mbsto Indurido } \\
\text { (casos) }\end{array}$}} & \multicolumn{2}{|c|}{ Gestagajo a Terms } & \multirow{3}{*}{ CA $(\mathrm{CO})$} & \multirow{3}{*}{ p-valor } \\
\hline & & & & & & \\
\hline & N & $\%$ & N & $\%$ & & \\
\hline \multicolumn{7}{|l|}{ Idade } \\
\hline $14-19$ & 4 & 19.0 & 23 & 27,7 & & \\
\hline $20-24$ & 8 & 38,1 & 29 & 34.9 & & \\
\hline $25-30$ & 6 & 28,6 & 22 & 26.5 & 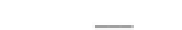 & $P=0,86$ \\
\hline 30 e mais & 3 & 14,3 & 9 & 109 & & \\
\hline Total & 21 & 100,0 & 83 & 100 & & \\
\hline \multicolumn{7}{|l|}{ Cor da pele } \\
\hline Branca & 3 & 14,3 & 18 & 21,7 & $O R=0,60$ & $P=0,55$ \\
\hline Näo branta & 18 & 85,7 & 65 & 78,3 & $(0,13-2,53)$ & \\
\hline Toal & 21 & 100 & 83 & 100 & & \\
\hline \multicolumn{7}{|l|}{ Anos de estudo } \\
\hline$<o u$ igud a 7 & 8 & 38,1 & 41 & 49,4 & $O R=0,63$ & $\mathrm{P}=0,35$ \\
\hline$<7$ & 13 & 61,9 & 42 & 50.6 & $(0.21-1.85)$ & \\
\hline Total & 21 & 100 & 83 & 100 & & \\
\hline \multicolumn{7}{|l|}{ Religia } \\
\hline Catúlica & 9 & 42,9 & 27 & 32.5 & & \\
\hline Evangdica & 9 & 42,9 & 44 & 53.0 & 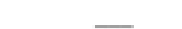 & $\mathrm{P}=0,65$ \\
\hline Nerhuma & 3 & 142 & 12 & 14.5 & & \\
\hline Total & 21 & 100 & 83 & 100 & & \\
\hline \multicolumn{7}{|l|}{ Pratica a religiäo } \\
\hline Sim & 8 & 44,4 & 39 & 54.9 & $O A=0,66$ & $P=0,42$ \\
\hline Näo & 10 & 55.6 & 32 & 45,1 & $(0.21-2.06)$ & \\
\hline Total & 18 & 100 & $\pi 1$ & 100 & & \\
\hline $\begin{array}{l}\text { Renda mensal (salirio } \\
\text { mírieno) }\end{array}$ & & & & & & \\
\hline$<\alpha$ igud a 3 & 13 & 61,9 & 62 & 74.7 & $O A=0,55$ & $\mathrm{P}=0,24$ \\
\hline$<3$ & 8 & 38,1 & 21 & 25.3 & $(0.18-1,70)$ & \\
\hline Tocal & 21 & 100 & 83 & 100 & & \\
\hline \multicolumn{7}{|l|}{ Estado coniugal } \\
\hline \multicolumn{7}{|l|}{ Casadal en uniass estavet } \\
\hline Nanoranda/ separada/' & 5 & 23,8 & 67 & 807 & & \\
\hline divorciada/ solteira & & & & & OH $=0,07$ & $\mathrm{P}=0,00$ \\
\hline \multirow[t]{3}{*}{ Total } & & & & & $(0.02-0.26)$ & \\
\hline & 16 & 762 & 16 & 19.3 & & \\
\hline & 21 & 100 & 83 & 100 & & \\
\hline \multicolumn{7}{|l|}{$\begin{array}{l}\text { Ocupaşoi } \\
\text { protsstio }\end{array}$} \\
\hline Desempregadalestudandoitra & & & & & $\mathrm{OA}=7,50$ & $\mathrm{P}=0,00$ \\
\hline balhando tora & & & & & {$[2,31-24,4\}$} & \\
\hline Dona de casa & 17 & 81,0 & 30 & 36,1 & & \\
\hline \multirow[t]{2}{*}{ Told } & 4 & 19.0 & 53 & 63.9 & & \\
\hline & 21 & 100 & 83 & 100 & & \\
\hline
\end{tabular}


Em relação às características reprodutivas, não houve diferenças significativas entre os grupos em relação à idade da menarca, idade da coitarca, idade da primeira gestação, número de gestações, intervalo entre a última e penúltima gestação e 0 número de filhos vivos (Tabela 2).

Todas as mulheres do grupo abortamento induzido referiram não ter planejado a gestação, contra $74,7 \%$ do grupo-controle. No grupo-controle, $77,1 \%$ das mulheres referiram ter desejado a gravidez. Nota-se que declararam desejo pela gestação quatro entrevistadas pertencentes ao grupo abortamento induzido.

As correlações realizadas entre os grupos abortamento induzido e o controle (Tabela 2) demonstram diferenças significativas para desejo pela gestação. 0 grupo que induziu o abortamento tinha 14,316 vezes mais chances de não desejar a gravidez.

\begin{tabular}{|c|c|c|c|c|c|c|}
\hline \multirow[b]{3}{*}{ Caracteristicas } & \multirow{2}{*}{\multicolumn{2}{|c|}{$\begin{array}{l}\text { Aborto Induzido } \\
\text { (casos) }\end{array}$}} & \multirow{2}{*}{\multicolumn{2}{|c|}{$\begin{array}{l}\text { Gestaçäo a Termo } \\
\text { (controles) }\end{array}$}} & \multirow{3}{*}{ OR $(I C)$} & \multirow{3}{*}{ p-valor } \\
\hline & & & & & & \\
\hline & $N$ & $\%$ & $N$ & $\%$ & & \\
\hline \multicolumn{7}{|l|}{ Menarca } \\
\hline $9-12$ & 10 & 47,6 & 42 & 50,6 & & \\
\hline $13-14$ & 8 & 38,1 & 37 & 44,6 & - & $\mathrm{P}=0,188$ \\
\hline 15 e mas & 2 & 9,5 & 4 & 4,8 & & \\
\hline Näo soube informar & 1 & 4,8 & 0 & 0 & & \\
\hline Total & 21 & 100 & 83 & 100 & & \\
\hline \multicolumn{7}{|l|}{ Coitarca } \\
\hline $12-15$ & 7 & 33,3 & 39 & 47,0 & $O R=0,56$ & $P=0,26$ \\
\hline $16 \mathrm{e}$ mais & 14 & 66,7 & 44 & 53,0 & $(0,18-1,70)$ & \\
\hline Total & 21 & 100 & 83 & 100 & & \\
\hline \multicolumn{7}{|l|}{ Idade da $1^{3}$ gestaçāo } \\
\hline $13-19$ & 13 & 61,9 & 53 & 63,9 & $\mathrm{OR}=0,92$ & $P=0,87$ \\
\hline $20 \mathrm{e}$ mais & 8 & 38,1 & 30 & 36,1 & $(0,31-2,77)$ & \\
\hline Total & 21 & 100 & 83 & 100 & & \\
\hline \multicolumn{7}{|l|}{ Gestaçõoes } \\
\hline $1-2$ & 11 & 52,4 & 50 & 60,2 & $O R=0,73$ & $P=0,51$ \\
\hline 3 ou mais & 10 & 47,6 & 33 & 39,8 & $(0,25-2,11)$ & \\
\hline Total & 21 & 100 & 83 & 100 & & \\
\hline \multicolumn{7}{|c|}{$\begin{array}{l}\text { Intervalo entre a óltima e penúltima } \\
\text { gestação (anos) }\end{array}$} \\
\hline \multicolumn{7}{|c|}{2 e menos } \\
\hline $3 \mathrm{e}$ mas & 6 & 54,5 & 24 & 44,4 & $O R=1,5$ & $P=0,74$ \\
\hline \multirow[t]{2}{*}{ Total } & 5 & 45,5 & 30 & 55,6 & $(0,33-6,99)$ & \\
\hline & 11 & 100 & 54 & 100 & & \\
\hline \multicolumn{7}{|l|}{ Número de filhos vivos } \\
\hline \multicolumn{7}{|l|}{1 e menos } \\
\hline 2 e mais & 12 & 57,1 & 54 & 65,1 & $O R=0,72$ & $P=0,50$ \\
\hline \multirow[t]{2}{*}{ Total } & 9 & 42,9 & 29 & 34,9 & $(0,24-2,11)$ & \\
\hline & 21 & 100 & 83 & 100 & & \\
\hline \multicolumn{7}{|l|}{ Gravidez planejada } \\
\hline Sim & 0 & 0 & 21 & 25,3 & - & $P=0,01$ \\
\hline Năo & 21 & 100 & 62 & 74,7 & & \\
\hline Total & 21 & 100 & 83 & 100 & & \\
\hline \multicolumn{7}{|l|}{ Gravidez desejada } \\
\hline Sim & 4 & 19,0 & 64 & 77,1 & $O R=14,32$ & $P=0,00$ \\
\hline Năo & 17 & 81,0 & 19 & 22,9 & $(4,297-47,699)$ & \\
\hline Total & 21 & 100 & 83 & 100 & & \\
\hline
\end{tabular}

A Tabela 3 traz informações a respeito do conhecimento sobre o Programa de Planejamento Familiar do Município da Serra, o recebimento de orientações sobre o planejamento da família, o uso de métodos contraceptivos na época da concepção e se alguém aconselhou as mulheres entrevistadas a abortar.

0 Programa de Planejamento Familiar do Município não é conhecido pela maioria das entrevistadas dos dois grupos; entretanto, as mulheres que induziram o abortamento foram as que, proporcionalmente, referiram maior desconhecimento
$(85,7 \%)$, fato esse que representou diferença estatística, $(p=0,06)$. Quando perguntadas se receberam algum tipo de orientação sobre o tema planejamento familiar no decorrer da vida, menos da metade das mulheres dos dois grupos declarou ter tido esse tipo de orientação: $42,9 \%$ do grupo abortamento induzido e $44,6 \%$ do grupo-controle $(p=0,88)$.

Grande parte das entrevistadas dos dois grupos não utilizava método anticoncepcional quando engravidou: $47,6 \%$ do grupo abortamento induzido e $61,4 \%$ do grupo-controle. No grupo- 
controle, o fato de querer engravidar apareceu como principal motivo para o não-uso de método anticoncepcional, em 45,1\% das respostas, seguido de efeitos colaterais indesejáveis $(9,8 \%)$ e não gostar de usar métodos anticoncepcionais $(9,8 \%)$. Outros motivos mencionados foram: achar que não podia engravidar, não ter dinheiro para comprar a pílula, não saber como tomar a pílula, não ter tempo para assistir a palestra na unidade de saúde, estar amamentando e estar com parceiro fixo. No grupo abortamento provocado, os principais motivos foram: não ter relação sexual com freqüência (40\%) e efeitos colaterais indesejáveis (20\%).

Das mulheres do grupo-controle que utilizavam algum método anticoncepcional quando engravidaram, a maioria estava em uso de pílula, 90,6\%. As entrevistadas do grupo abortamento induzido referiram com maior freqüência $(54,5 \%)$ o uso de preservativo. Quando perguntadas por que engravidaram mesmo utilizando método anticoncepcional, as respostas mais freqüentes foram esquecimento do uso da pílula e uso inadequado do preservativo.

Em relação à indicação do método anticoncepcional, 62,4\% das mulheres, dos dois grupos, disseram ter sido orientadas quanto ao uso do método anticoncepcional pelo ginecologista, $19 \%$ por ninguém, $13,4 \%$ por amigos e familiares, $4,2 \%$ por balconista de farmácia e $1 \%$ pela assistente social.

Das mulheres que interromperam a gravidez, $47,6 \%$ delas foram aconselhadas a abortar e um pequeno percentual do outro grupo recebeu o mesmo conselho, 24,1\%. Quando comparados abortamento induzido e grupo-controle, em relação a se alguém aconselhou a abortar, as diferenças foram significantes $(P=0,03)$.

Tabela 3 - Distribuiçāo dos grupos segundo variáveis relocionadas ao planejamento familiar e conselho em relação ao abortamenta. Maternidade de Carapina - Serra - $1^{\circ}$ de agosto de 2005 a 31 de janeiro de 2006

\begin{tabular}{|c|c|c|c|c|c|c|}
\hline \multirow[b]{2}{*}{ Caracteristicas } & \multicolumn{2}{|c|}{$\begin{array}{l}\text { Aborto Induzido } \\
\text { (cosos })\end{array}$} & \multicolumn{2}{|c|}{$\begin{array}{c}\text { Gestoçóo a Termo } \\
\text { (controles) }\end{array}$} & \multirow[t]{2}{*}{ OR (IC) } & \multirow[t]{2}{*}{ p-wolor } \\
\hline & $\mathrm{N}$ & $\%$ & $\mathrm{~N}$ & $\%$ & & \\
\hline $\begin{array}{l}\text { Conhecimento do programo de } \\
\text { plonejomento fomilior do município } \\
\text { Sim } \\
\text { Nōo } \\
\text { Totol }\end{array}$ & $\begin{array}{r}3 \\
18 \\
21 \\
\end{array}$ & $\begin{array}{r}14,3 \\
85,7 \\
100 \\
\end{array}$ & $\begin{array}{l}31 \\
52 \\
83 \\
\end{array}$ & $\begin{array}{l}37,3 \\
62,7 \\
100 \\
\end{array}$ & $\begin{array}{c}O R=0,28 \\
(0,05-1,08)\end{array}$ & $P=0,06$ \\
\hline $\begin{array}{l}\text { Reccebimento de orientaçóo sobre o } \\
\text { planejamento fomilior } \\
\text { Sim } \\
\text { Não } \\
\text { Total }\end{array}$ & $\begin{array}{c}9 \\
12 \\
21 \\
\end{array}$ & $\begin{array}{l}42,9 \\
57,1 \\
100 \\
\end{array}$ & $\begin{array}{l}37 \\
46 \\
83 \\
\end{array}$ & $\begin{array}{l}44,6 \\
55,4 \\
100 \\
\end{array}$ & $\begin{array}{l}\text { OR }-0,93 \\
\{0,32-2,71]\end{array}$ & $P=0,8 B$ \\
\hline $\begin{array}{l}\text { Utilização de método anticoncepcional } \\
\text { na época da concepçóo } \\
\text { Sim } \\
\text { Nōo } \\
\text { Total }\end{array}$ & $\begin{array}{l}11 \\
10 \\
21 \\
\end{array}$ & $\begin{array}{r}52,4 \\
47,6 \\
100 \\
\end{array}$ & $\begin{array}{l}32 \\
51 \\
83 \\
\end{array}$ & $\begin{array}{r}38,6 \\
61,4 \\
100 \\
\end{array}$ & $\begin{array}{l}\mathrm{OR}=1,75 \\
{[0,61-5,11]}\end{array}$ & $P=0,25$ \\
\hline $\begin{array}{l}\text { Ter sido oconsel hodo o obortor } \\
\text { Sim } \\
\text { Não } \\
\text { Totol }\end{array}$ & $\begin{array}{l}10 \\
11 \\
21\end{array}$ & $\begin{array}{l}47,6 \\
52,4 \\
100 \\
\end{array}$ & $\begin{array}{l}20 \\
63 \\
83 \\
\end{array}$ & $\begin{array}{l}24,1 \\
75,9 \\
100 \\
\end{array}$ & $\begin{array}{l}\text { OR }-2,86 \\
(0,92-8,63)\end{array}$ & $P=0,03$ \\
\hline
\end{tabular}

Para avaliar o efeito conjunto, recíproco e simultâneo das variáveis que por meio da análise bivariada demonstraram significância para a indução do abortamento, um modelo de regressão logística foi adicionado. No modelo proposto na Tabela 4, as únicas variáveis que apresentaram valores significativos foram: estado conjugal casada/em união estável $(p=0,042)$ e desejo pela gestação $(p=0,011)$.

Tabela 4 - Análise de regressão logística. Maternidade de Carapina - Serra - $1^{\circ}$ de agosto de 2005 a 31 de janeiro de 2006

\begin{tabular}{|c|c|c|c|c|}
\hline Modelo & Variável & OR & $\mathrm{IC} \mathrm{a} 95 \%$ & $\mathrm{P}$ \\
\hline \multirow{6}{*}{$\begin{array}{l}1 \text { - Identificoçōo } \\
\text { (abortamento induzido e } \\
\text { gestoçáo a termo) + } \\
\text { estodo conjugol + } \\
\text { ocupoçōo + } \\
\text { planejamento da gravidez + } \\
\text { desejo pela gestação }+ \\
\text { conhecer o Programa de } \\
\text { Planejomento Fomilior }+ \\
\text { ter sido aconselhada a } \\
\text { abortar }\end{array}$} & Estado coniugal & 0,241 & $0,061-0,951$ & 0,042 \\
\hline & Ocupoçбó & 0,404 & $0,095-1,735$ & 0,223 \\
\hline & Plonejomento do grovidez & 0,534 & $0,045-6,339$ & 0,619 \\
\hline & Desejo pela grovidez & 0,168 & $0,042-0,669$ & 0,011 \\
\hline & $\begin{array}{l}\text { Conhecer o Programa de } \\
\text { Planejamento Fomiliar }\end{array}$ & 0,559 & $0,115 \cdot 2.706$ & 0,470 \\
\hline & $\begin{array}{l}\text { Ter sido aconselhada a } \\
\text { abortor }\end{array}$ & 2,233 & $0,619-8,056$ & 0,220 \\
\hline
\end{tabular}

LR chi $2(4)-38,47$ Prob $>$ chi2 $=0,000$ 


\section{DISCUSSÃO}

0 abortamento induzido, como grave problema de saúde pública, traz prejuízos para a sociedade, principalmente em relação a sua contribuição para o aumento da mortalidade materna, e investigar os fatores relacionados à sua prática é de suma importância para orientar políticas públicas que visem à redução dos casos de abortamento inseguro e, conseqüentemente, a diminuição da mor talidade materna.

Este estudo teve como objetivo comparar aspectos sociodemográficos e reprodutivos entre mulheres que induziram a interrupção da gestação e mulheres que levaram a gestação a termo admitidas em uma maternidade pública. 0 estado conjugal casada/em união estável e o desejo pela gravidez foram as únicas variáveis que se mostraram associadas, como fatores de proteção, à ocorrência de abortamento induzido.

Como limitações do estudo com poder de subestimar as associações encontradas destacam-se: o fato de que, possivelmente, algumas mulheres não revelaram a verdadeira causa da interrupção da gestação e declararam o abortamento como espontâneo; o não-conhecimento da proporção total dos abortamentos induzidos no Município da Serra que requerem hospitalização; e o viés de memória, pois muitas informações perguntadas às entrevistadas exigiam a lembrança de fatos passados.

0 desejo pela gestação como fator de proteção para a indução do abortamento está totalmente relacionado com o planejamento da gravidez, pois a partir do momento que a mulher, ou melhor, que o casal decide ter um filho, essa decisão é encorajada pelo desejo de desempenhar o papel de pai e mãe. A aceitação de uma gestação não planejada se torna mais complicada, principalmente, quando não há estabilidade conjugal, o que é comprovado pelo resultado apresentado na presente pesquisa e relatada em outros estudos ${ }^{11,12}$.

A eventualidade e o não-planejamento das relações sexuais, geralmente associadas aos relacionamentos instáveis, influenciam diretamente no não-uso de métodos contraceptivos pelas mulheres, em conjunto com o medo dos efeitos colaterais ${ }^{4}$. Essa afirmação reforça a necessidade da população em relação a uma educação que abranja as informações relativas ao planejamento da família e, provavelmente, também, à sexualidade, às relações de gênero, às doenças sexualmente transmissíveis e a outros assuntos que, de maneira geral, ainda são tratados como tabus pela sociedade.

Em relação à situação conjugal, os achados da pesquisa estão em concordância com os encontrados em outros estudos em que uma baixa proporção de mulheres que induziram 0 abortamento eram casadas ${ }^{11,12}$. As conclusões do estudo de Cates Júnior, Grimes e Schulz ${ }^{13}$ revelam que a proporção de mulheres solteiras que provocam o abortamento nos Estados Unidos cresceu, entre 1972 e 1999, de 70,0\% para 80,0\%. Para se ter uma idéia de como a situação conjugal influencia na decisão de abortar ou não, independente do status socioeconômico do país de moradia, o levantamento sobre os motivos que levam mulheres a abortar, feito, também nos Estados Unidos, por Finer et al. ${ }^{14}$, revela que mais da metade das mulheres citaram como razão problemas no relacionamento ou não querer ser mãe solteira. 0 fato de a mulher ser casada oferecer uma proteção de $93 \%$ contra o abortamento provocado é confirmado pelo estudo de Lucena ${ }^{15}$, que, ao comparar abortamento espontâneo com gestação a termo, conclui que as mulheres solteiras têm risco duas vezes maior para realizar 0 abortamento induzido.

Torna-se imprescindível refletir sobre a participação do homem nas questões ligadas à saúde sexual e reprodutiva e sobre a importância da paternidade responsável e da valorização da família.

$\mathrm{Na}$ Conferência Internacional sobre População e Desenvolvimento, realizada no Cairo, em 1994, e na IV Conferência Mundial sobre Mulheres, realizada em Beijing, em 1995, teve início a discussão a respeito da influência dos padrões de comportamento sexual e reprodutivo do homem sobre indicadores desfavoráveis de saúde da mulher, valorizando a importância da responsabilidade individual e social masculina ${ }^{16}$. Mais de dez anos se passaram e nota-se que a participação dos homens nos processos relativos à sexualidade e à reprodução ainda precisa, cada vez mais, ser incentivada e valorizada.

Em pesquisa realizada com os funcionários de um hospital filantrópico sobre a participação do homem na saúde reprodutiva, observa-se que os relatos dizem que a presença masculina é estimulada em todos os setores hospitalares, mas é vista com menos freqüência no local reservado para as mulheres no pós-aborto ${ }^{16}$.

Estudo com jovens de diferentes estratos sociais, entre 18 e 24 anos, revelou que os homens declaram com maior facilidade a realização do abortamento por parte da parceira, " [...] demonstrando uma expressiva presença da idéia desse recurso face à gravidez não prevista [...]". Os autores explicam esse achado devido ao fato de que os homens muitas vezes não têm certeza da paternidade e até mesmo da prática do abortamento vivenciado pela mulher ${ }^{17: 1418}$, o que, mais uma vez, confirma o posicionamento do parceiro no processo de tomada de decisão pela manutenção da gravidez e responsabilização de seu papel como pai.

Também merece ser citada a desvalorização de padrões sociais em que a formação de uma família com filhos estava vinculada ao casamento e à estabilidade conjugal. Mesmo as mulheres tendo um maior envolvimento com as conseqüências de seus atos em relação à sexualidade e à reprodução, enquanto os homens assumem uma postura menos compromissada, quando elas se deparam com uma gestação não planejada, principalmente se tratando de adolescentes, necessitam, em sua maioria, da ajuda dos pais, parceiros e familiares ${ }^{18}$. E nesse caso, a valorização da estabilidade conjugal e da constituição de uma família é de suma importância para a saúde sexual e reprodutiva de homens e mulheres e para a prevenção de abortamentos inseguros. 


\section{CONCLUSÃO}

A falta de estabilidade marital e de desejo pela gravidez como fatores influenciadores da opção pelo abortamento deixa claro que essas mulheres não escolheram o melhor momento, $\mathrm{e}$ em alguns casos, também, o parceiro certo, para se ter um filho, revelando o problema da ausência de planejamento em relação às suas vidas reprodutivas.

Quando se fala em planejamento familiar, a tendência é planejar ações voltadas, principalmente, para o público feminino, esquecendo-se de se trabalhar com o conceito de paternidade responsável. Através da conclusão dessa pesquisa fica claro que a falta de apoio do companheiro é fator determinante para a indução do abortamento; dessa maneira, para tentar diminuir

\section{Referências}

1. Schor N, Alvarenga AT. 0 aborto: um resgate histórico e outros dados. Rev Bras Crescimento Desenvolv Hum 1994; 4(2): $12-7$.

2. Rocha MIB, Andalaft Neto J. A questão do aborto: aspectos clínicos, legislativos e políticos. In: Berquó E, organizadora. Sexo e vida: panorama da saúde reprodutiva no Brasil. Campinas (SP): Ed UNICAMP; 2003. p. 257 - 318.

3. Faúndes A, Duarte GA, Osis MJD, Andalaft Neto J. Variação no conhecimento e nas opiniões dos ginecologistas e obstetras brasileiros sobre o aborto legal entre 2003 e 2005. Rev Bras Ginecol Obstet 2007; 29 (4): 192-99.

4. Sorrentino S. Aborto inseguro. In: Rede Nacional Feminista de Saúde e Direitos Reprodutivos. Saúde da mulher e direitos reprodutivos: dossiês. São Paulo (SP); 2001. p. 41 - 72.

5. Billings D, Benson J. Postabortion care in Latin América: policy and service recommendations from a decade of operations research. London: London(UK): Oxford University Press/ School of Hygiene and Tropical Medicine; 2005. p. 158- 66.

6. Dallari SG. Aborto um problema ético da saúde pública. Bioética 1994; 2 (1): 37- 41.

7. Nader PRA, Blandino VRP, Maciel ELN. Características de abortamentos atendidos em uma maternidade pública do Município da Serra (ES). Rev Bras Epidemiol 2007 dez; 10 (4): 615-24.

8. Prefeitura Municipal da Serra (ES). Secretaria Municipal de Saúde. Sistema de Informação de Mortalidade. Sistema de Informação de Nascidos Vivos. Mortalidade materna. Serra (ES); 2004.

9.World Health Organization-WHO. Protocol for hospital based descriptive studies of mortality, morbidity related to induced abortion. Geneva(CH): WHO taste force on safetyand efficacy of fertility regulating methods, 1987. o número de abortamentos induzidos, é fundamental que se comecem a discutir ações voltadas para a conscientização e responsabilidade masculina em relação ao planejamento da família.

Pelo exposto, prevenir o abortamento inseguro, evitando a ocorrência de conseqüências graves para a vida afetiva, biopsíquica e social da população feminina, é sinônimo de investir em promoção à saúde reprodutiva e em melhores condições de vida para a população. Ações relacionadas com a orientação sexual e contracepção com uma abordagem que facilite a discussão de aspectos culturais e psicológicos, promovendo reflexão, devem ser incentivadas e acessíveis para mulheres e homens e, principalmente, para adolescentes.

10. Fonseca W, Misago C, Freitas P, Santos E, Fernandes L, Correia L. Características sócio-demográficas, reprodutivas e médicas de muIheres admitidas por aborto em hospital da Região Sul do Brasil. Cad Saude Publica, 1998 abr/jun; 14 ( 2): 279-86.

11. Kac G, Silveira EA, Oliveira LC, Araujo DMR, Souza EB. Fatores associados a ocorrência de cesárea e aborto em mulheres selecionadas em um centro de saúde no município do Rio de Janeiro, Brasil. Rev Bras Saude Matern Infant 2007 jul/set; 7 (3): 271-80.

12. Fusco CLB, Andreoni S, Souza e Silva R. Epidemiologia do abor to inseguro em uma população em situação de pobreza - favela Inajar de Souza, São Paulo. Rev Bras Epidemiol 2008; 11 (1): 78-88.

13. Cates Junior W, Grimes DA, Schulz KF. The public health impact of legal abortion: 30 years later. Perspect Sex Reprod Health 2003; 35: 25-8.

14. Finer LB, Frohwirth LF, Dauphinee LA, Singh S, Moore AM. Reasons U. S. women have abortions: quantitative and qualitative perspectives. Perspect Sex Reprod Health 2005; 37: 110-18.

15. Lucena RCB. Características de mulheres internadas por aborto em uma maternidade pública em Recife (PE): dimensão do problema e sua relação com a prática contraceptiva. [dissertação de mestrado] Recife (PE): Centro de Pesquisa Aggeu Magalhães /Núcleo de Estudos em Saúde Coletiva/ FIOCRUZ; 2000.

16. Galastro EP, Fonseca RMGS. A participação do homem na saúde reprodutiva: o que pensam os profissionais de saúde. Rev Esc Enferm USP 2007; 41 (3): 454-59.

17. Peres SO, Heilborn ML. Cogitação e prática do aborto entre jovens em contexto de interdição legal: o avesso da gravidez na adolescência. Cad Saude Publica 2006 jul; 22 (7): 1411-20.

18. Arcanjo CM, Oliveira MIV, Bezerra MGA. Gravidez em adolescentes de uma unidade municipal de saúde em Fortaleza (CE). Esc Anna Nery Rev Enferm 2007 set; 11(3): 445-51. 